\title{
Patterns of interocular transfer of visuomotor coordination reveal differences in the representation of visual space
}

\author{
V. A. MANN, A. HEIN, and R. DIAMOND \\ Massachusetts Institute of Technology, Cambridge, Massachusetts 02139
}

\begin{abstract}
Acquisition and interocular transfer of adaptation to optical transformations of input were examined in strabismic and orthotropic subjects. Distinct patterns of behavior were displayed by three groups: strabismic alternating suppressors, strabismic and orthotropic constant suppressors, and orthotropes with normal binocularity of vision. It is suggested that these behaviors result from the way in which visual space is represented in each group, an outcome of the pattern of use of the eyes during early development. The conditions for modification of the representation of visual space appear to parallel those for initial acquisition.
\end{abstract}

Two principal types of monocular suppression are found among strabismic individuals: an alternating form, in which vision in each eye is suppressed part of the time, and a constant form, in which vision in one eye is consistently suppressed. We recently examined visually guided reaching in strabismic individuals and in persons with normal binocularity of vision (Mann, Hein, \& Diamond, 1979). Like persons in whom the two eyes converge at the same point in visual space, alternating suppressors localized targets equivalently when using either eye, while constant suppressors did not. The discrepancies in target localization displayed by constant suppressors were attributed to their utilization of information about the posture of the dominant eye in localizing targets viewed monocularly by either eye. This implies that when targets are viewed by the normally suppressed eye, retinal locus information from that eye is combined with postural information from the dominant eye. The necessity for constant suppressors to rely upon information about the posture of the occluded eye was viewed as a result of the way in which a representation of visual space had been acquired during early development. This representation is assumed to incorporate the correspondence between direction of objects in space, retinal loci of images, and the set of postures that the eye can assume. Constant suppression of vision in one eye

This research was supported by N.I.H. Grant 5-R01-NF09279 to Dr. A. Hein, by a grant from the Spencer Foundation to Dr. H.-L. Teuber, and by a National Science Foundation Graduate Traineeship to the senior author. We thank Dr. R. Held for providing the goggles used for optical rotation of the visual field, and Dr. J. Bauer for his assistance with setting up the apparatus used in Experiment 1. We also thank Dr. J. Rosner and the staff of the Pediatric Clinic of the New England College of Optometry for their cooperation in obtaining subjects. Reprint requests should be addressed to: V. A. Mann, Haskins Laboratories, Inc., 270 Crown Street, New Haven, Connecticut 06510. was hypothesized to permit acquisition of a representation of visual space only with regard to information from the dominant eye.

Among higher mammals, visually guided behavior develops during exposure in light (Riesen, 1958). A series of studies with kittens has indicated that, if the two eyes are exposed separately, each may independently acquire the capacity to mediate guided movements (Dews \& Wiesel, 1970; Ganz \& Fitch, 1968; Hein \& Diamond, 1971, 1972; Hein, Held \& Gower, 1970). It appeared possible that, in humans, the outcome of alternating suppression of vision might resemble that in kittens reared with alternating monocular occlusion. Thus, strabismics in whom each eye is alternately suppressed may have independently acquired the capacity to guide movements with each eye. The representations of visual space which support these behaviors would have been formed under a condition in which vision was effectively monocular. The correspondence between object direction and eye posture and locus of retinal images for each eye would therefore have been incorporated into a separate representation of visual space. However, since each eye would have been used under comparable circumstances, these monocular representations should be equivalent. Consistent with this, alternating suppressors did not differ from orthotropes in localizing targets presented to monocular view (Mann, Hein, \& Diamond, 1979).

In the cat, if each eye is exposed under conditions which differ with regard to opportunities for motorvisual feedback, the components of visually guided behavior mediated by each eye will also differ (Hein \& Diamond, 1971, 1972). Lack of interocular transfer of visuomotor capacities in these animals suggests a means to evaluate the status of human alternating suppressors. If coordination is modified in these persons under conditions of monocular 
exposure, the modification may be absent when behavior is mediated by the unexposed eye. In this respect, alternating suppressors should differ from persons with normal binocular vision. It is assumed that during binocular vision the posture of both eyes in relation to the loci of images on both retinae provides information about the direction of objects. This account is in agreement with a report by Ono, Wilkinson, Muter, and Mitson (1972) that in normal orthotropes the position of both eyes influences both monocular and binocular judgements of direction. Normal binocular vision during early development should produce a single representation of visual space common to both eyes. Our description implies that persons with normal binocularity of vision, in whom coordination is modified during monocular exposure, will display the modification when tested using the fellow eye as well.

Previous studies have provided evidence that the mechanism which underlies initial acquisition of visually guided behaviors also operates in adjustment of coordination to prismatic displacement of visual input (Held \& Bossom, 1961; Held \& Hein, 1963). Prismatic displacement of input changes the relation between the direction of targets and the retinal loci of their images. Adjustment of coordinated movement to this changed relationship demands that the new correspondence between eye postures, image loci, and object direction be taken into account. In effect, this is a revision of the original representation of visual space. This line of reasoning suggests that persons with normal binocularity, alternating suppressors, and constant suppressors will differ in the acquisition and interocular transfer of adaptation to prismatic displacement of input. Persons with normal binocularity of vision should adapt when displaced input is provided to either eye, and the resulting compensatory shift in coordination should be mediated by the unexposed eye as well. Alternating suppressors should adàpt when displaced input is provided to either eye, but should compensate only when using the eye that has been exposed. The behavior of constant suppressors should depend upon whether the dominant eye or the normally suppressed eye is exposed. Exposure of the normally suppressed eye with displaced input should not produce adaptation. This follows from the model we have proposed, because orbital postures and loci of images on the retina of the suppressed eye have not entered into a representation of visual space. When the dominant eye is exposed with displaced input, the representation of visual space which incorporated information about that eye should be revised and adaptation should result. Moreover, when the subject is tested using the unexposed, normally suppressed, eye, the revised representation should control behavior. This outcome results from the same dependence according to which the original representation related to the dominant eye influenced reaches mediated by the normally suppressed eye (Mann, Hein, \& Diamond, 1979).

In individuals with normal binocularity of vision, adaptation of visuomotor coordination to prismatic displacement of input has been found to transfer interocularly (Crawshaw \& Craske, 1976; Ebenholtz, 1967; Hajos \& Ritter, 1965; Mack \& Chityat, 1970). The issue had not yet been addressed in strabismic subjects. Experiment 1 examined the hypothesis outlined above. Strabismics in whom vision in each eye is alternately suppressed, strabismics and orthotropes in whom vision in one eye is constantly suppressed, and orthotropes with normal binocularity of vision participated. Each subject viewed self-produced arm movements while a laterally displacing prism was worn in front of one eye. The dominant eye was exposed in one session and the nondominant eye in another. Adaptation was measured as a postexposure shift in the direction of reaches and was tested for both the exposed and the unexposed eye in each session. Experiment 2 extended the same logic to an examination of the effects of optical rotation of the visual field. Adaptation was assessed by postexposure shifts in setting a grating to apparent vertical.

\section{EXPERIMENT 1}

\section{Subjects}

\section{Method}

Sixteen strabismic individuals, eight orthotropes with normal binocular vision, and four amblyopic orthotropes participated. Strabismic subjects and amblyopic orthotropes were referred through the pediatric clinic of the New England College of Optometry and by optometrists in private practice in the Boston area. Strabismic individuals included eight alternating suppressors (four esotropes and four exotropes) and eight constant suppressors (four esotropes and four exotropes). In all cases, a marked horizontal error of vergence had been noted by parents or clinicians prior to age 1 . None of the strabismic subjects displayed abnormal retinal correspondence, as revealed by a standard clinical test employing afterimages (Hansen, 1954).

The four amblyopic orthotropes included three persons with marked monocular astigmatism and one with aniseikonia. Each of these persons constantly suppressed vision in the nondominant eye.

Evaluation of visual status. Where available, clinical records provided preliminary information about visual acuity, stereoacuity, eye dominance, vergence error, and pattern of use of the eyes. This information was supplemented and confirmed by tests of visual functioning conducted at the beginning of the first experimental session. If the subject normally wore corrective lenses, they were removed for this evaluation and for subsequent testing. Visual acuity was evaluated with a Snellen chart located $6.1 \mathrm{~m}$ from the viewer. Stereoacuity was determined with a test using Julesz random-dot stereograms (Reinecke \& Simon, 1974). Following standard clinical practice, the dominant eye was defined as the eye used to direct the preferred hand toward a visual target with both eyes open. Subjects were classified as strabismic or nonstrabismic (orthotropic) using a standard clinical cover test reported sensitive to vergence errors of 4 diopters (Romano 
\& von Noorden, 1971). The difference in position of the images of a distant light source reflected on the two corneae confirmed the results of the cover test (Reinecke \& Miller, 1966).

Two tests were used to assess the pattern of use of the eyes. The first, a cover test, enabled strabismics to be classified as either constant or alternating suppressors. The subject attempts to maintain fixation of a visual target with one eye while the other eye is alternately occluded and uncovered. Subjects able to maintain fixation with either eye under these conditions were classified as alternating suppressors. Those unable to maintain fixation of a target with the nondominant eye when the dominant eye was uncovered were classified as constant suppressors (Reinecke \& Miller, 1966). Patterns of use were also evaluated with a modified Worth test in which colored stimuli and color filters permitted presentation of a separate monocular display to each eye (Burian \& von Noorden, 1974; Reinecke \& Miller, 1966). Subjects with binocular patterns of use on the cover test reported seeing the displays as superimposed. Alternating suppressors could report each monocular image but could not fuse the two. Constant suppressors reported only the display presented to the dominant eye unless that eye was occluded, in which case the display presented to the normally suppressed eye was reported.

\begin{abstract}
Apparatus
An apparatus modeled after that described by Held and Gottlieb (1958) was used, both to assess visually guided reaching and to provide exposure with prismatic displacement of input. For testing of visually guided reaching, the seated subject looked through an aperture at a half-silvered mirror oriented $45^{\circ}$ to the horizontal plane. The mirror prevented the subject from viewing his hand and arm while reflecting visual targets as virtual images which appeared to lie on a surface directly before him. The targets were four green-light-emitting diodes located $5^{\circ}$ and $20^{\circ}$ to the left and right of a central red-light-emitting diode which served as a constant reference point. For exposure with prismatic displacement of input, a 20-diopter Fresnel prism oriented base-in was placed in front of the viewing aperture. Target and reference lights were extinguished and the area below the surface of the half-silvered mirror illuminated, permitting the subject to view his hand and arm. An armrest brought the hand to eye level and restricted movement to $20^{\circ}$ of arc in a frontal plane.
\end{abstract}

\section{Procedure}

Hand-eye coordination was tested monocularly with each eye both before and after exposure of one eye with prismatic displacement of input. Using the method described by Bauer, Woods, and Held (1969), a rubber thimble with an electrode embedded in its tip was placed on the index finger of the subject's preferred hand. Contact of the electrode with a specifically prepared surface at the optical distance of the targets $(42.5 \mathrm{~cm})$ completed a circuit and caused the horizontal coordinate of the point of contact to be automatically computed.

Each trial began with the subject resting his arm in his lap. A target was illuminated; the subject was instructed to look at it, point to its apparent position with his index finger, and return his arm to his lap. Twelve practice trials with binocular view of the targets preceded monocular test trials. Six blocks of preexposure test trials were administered: the eye which would subsequently be exposed with prismatic displacement of input was used for Blocks 2, 4, and 6; the eye which would not be exposed was used for Blocks 1, 3, and 5. Each block consisted of 12 trials in which each of the four targets was presented three times in a fixed semirandom order. The subject wore a soft eye patch over the eye which was not being used. After each block of trials, he closed his eyes and transferred the patch to the other eye.

At the end of the final block of preexposure trials, the subject remained seated with eyes closed, wearing the eye patch over the same eye as for Block 6. His arm was placed in the holder, the area below the half-silvered mirror illuminated, and the prism positioned in the viewing aperture. The subject was then instructed to open his eye and view his arm while moving it right and left in time to a metronome at a rate of $30 \mathrm{cycles} / \mathrm{min}$. Ninety seconds of exposure alternated with $20 \mathrm{sec}$ of rest with eyes closed until the subject had viewed his arm for a total of $10 \mathrm{~min}$.

Immediately following the exposure period, hand-eye coordination was retested with the prism removed from the aperture. Adaptation to prismatic displacement of input produced a shift in the direction of reaches away from the side to which input was displaced. As in preexposure testing, six blocks of trials were administered: the subject used the eye which had not been exposed with prismatic displacement for Blocks 1,3 , and 5 and the eye which had been exposed for Blocks 2, 4, and 6 . If the centroids of markings mediated by the exposed eye were found to be displaced less than $2^{\circ}$ nasally, relative to the centroids of preexposure markings, the subject was given an additional 10-min period of exposure with the prism in front of the same eye. At the end of this second exposure period, hand-eye coordination was retested. For these cases, only data obtained following the second period of exposure are reported.

In a second experimental session, administered 4 to 7 days after the first, the entire procedure, including preexposure testing, exposure with prismatic displacement of input, and postexposure testing, was repeated. In this session, the fellow eye was uncovered during the exposure period. The session in which dominant and nondominant eye were exposed was counterbalanced among subjects within each subject group.

\section{Results}

\section{Variability of Markings}

For each subject, pre- and postexposure markings of each target mediated by each eye were analyzed separately for each experimental session. There were no significant differences in variance among targets, between pre- and postexposure tests, or between responses mediated by the dominant and nondominant eyes $(\mathrm{df}=11,11$; criterion values for permitting rejection of the null hypothesis at the .05 level are: $F<.3$ or $F>3.0$ ). This permitted a pooled variance to be computed for each subject $\left(s_{p}\right)$ for each experimental session. Mean value of $s_{p}$ was $2.1^{\circ}$; intersession and intersubject differences were not significant $(\mathrm{df}=88,88$; criterion values for rejecting the null hypothesis at the .05 level are: $F<.7$ or $F>1.4$ ).

\section{Differences Between Pre- and Postexposure Markings}

For each target, the centroid of pre- and postexposure markings mediated by each eye was determined. The shift following exposure was computed separately for each eye for the session in which the dominant eye was exposed and for the session in which the nondominant eye was exposed. Magnitudes of these differences were evaluated with t tests $(\mathrm{df}=$ 22; criterion values for rejecting the null hypothesis at the .05 level are: $|t|>1.7$ ). Averaging across targets yielded an estimate of the effect of exposure on markings mediated by the exposed and unexposed eye for each session. Mean values of the difference between pre- and postexposure markings for each eye (dominant or nondominant) for each condition 
(exposed or unexposed) are presented in Table 1. In each case, positive values reflect compensation for prismatic displacement of input.

\section{Normal Orthotropes}

All of these subjects showed adaptation to prismatic displacement of input when tested with the exposed eye. Shifts following exposure of the nondominant eye did not differ in magnitude from those following exposure of the dominant eye [sign test $r(8)=4, p<.25]$. The eye which had not been exposed with displaced input also mediated a shift regardless of whether the dominant or nondominant eye had been exposed. Shifts mediated by the unexposed eye did not differ in magnitude from those mediated by the exposed eye [sign test $\mathrm{r}(8)=3$, $\mathrm{p}<.25]$.

\section{Alternating Suppressors}

Following exposure of either eye with displaced input, these subjects displayed adaptation when tested using that eye. Shifts following exposure of the nondominant eye did not differ in magnitude from those following exposure of the dominant eye [sign test $\mathrm{r}(8)=4, \mathrm{p}<.25$ ]. However, compensation did not transfer to reaches mediated by the unexposed eye, for either eye.

\section{Constant Suppressors (Strabismic Constant Suppressors and Amblyopic Orthotropes)}

When the dominant eye was exposed with displaced input, eye-hand coordination mediated by that eye showed adjustment. Compensation transferred to reaches mediated by the unexposed eye with no loss in magnitude [sign test $\mathrm{r}(12)=5, \mathrm{p}<.25$ ]. However, when the normally suppressed eye was exposed with displaced input, adaptation failed to occur; the location of postexposure markings did not differ significantly from that of preexposure markings. This pattern of results was found uniformly among constant suppressors, whether strabismic or orthotropic. (All of these subjects, upon failing to display adaptation following a first period of exposure with the normally suppressed eye, had been provided an additional period of exposure of that eye as described in the Procedure section.)

\section{Discussion}

Both among orthotropes with normal binocularity of vision and among strabismic alternating suppressors, exposure of either eye with prismatic displacement of input produced an alteration in visually guided reaches mediated by that eye. For the orthotropes, this compensatory shift transferred to the unexposed eye. This is in agreement with previous observations (Crawshaw \& Craske, 1976; Ebenholtz, 1967; Hajos \& Ritter, 1965; Mack \&
Table 1 Adaptation and Interocular Transfer

\begin{tabular}{lccccc}
\hline & \multicolumn{2}{c}{$\begin{array}{c}\text { Exposed } \\
\text { Eye }\end{array}$} & & \multicolumn{2}{c}{$\begin{array}{c}\text { Nonexposed } \\
\text { Eye }\end{array}$} \\
\cline { 2 - 3 } \cline { 5 - 6 } & $\mathrm{D}$ & & $\mathrm{ND}$ & $\mathrm{D}$ & $\mathrm{ND}$ \\
\hline Normal & \multicolumn{4}{c}{ Orthotropes } \\
Amblyopic & 3.5 & & 3.3 & 3.4 & 3.1 \\
& 3.4 & & 0.5 & 0.7 & 3.5 \\
Alternating Suppressors & 3.5 & & 3.1 & 0.3 & 0.1 \\
Constant Suppressors & 3.9 & & 0.2 & -0.8 & 3.7 \\
\hline
\end{tabular}

Note-Values are expressed as the mean difference (in degrees) between pre-and postexposure markings. $D=$ dominant; $N D=$ nondominant.

Chityat, 1970). Alternating suppressors, on the other hand, failed to display compensation when tested using the unexposed eye. In contrast, both groups of constant suppressors (strabismics and amblyopic orthotropes) adapted to prismatic displacement of input only when the dominant eye was exposed. In these subjects, however, compensation did transfer to reaches mediated by the normally suppressed eye.

Visually guided reaching is only one component of visually coordinated behavior. In the kitten, the capacity to mediate guided movements of the forelimbs can be gained only after the animal has acquired the capacity to guide locomotory movements with respect to visual targets (Hein \& Diamond, 1972). Visually guided locomotion implies the existence of a body-centered representation of visual space within which objects, including the forelimbs, may be localized. Alternating suppressors, constant suppressors, and orthotropes with normal binocular vision have been hypothesized to differ with respect to the way in which visual space is represented. The behavior of these groups in tasks of target localization and in adapting to prismatic displacement of vision has been related to this model. All of these results, however, were obtained in tasks of hand-eye coordination. Differences among these subject groups in the way in which visual space is represented should be demonstrable in other contexts as well. It has been suggested that the coordinates of the inclusive body-centered representation of visual space are altered during adaptation to optical transformation of an extended visual field (Hein \& Diamond, 1972; Held \& Bossom, 1961; Held \& Mikaelian, 1964). Adjustment to this form of displaced input was studied in Experiment 2 among the subjects who had participated in Experiment 1. In separate sessions, the dominant and nondominant eye were exposed, with input optically rotated $20^{\circ}$ clockwise or counterclockwise. Adaptation was measured as a shift in visually mediated judgments 
of the vertical and was tested separately with the exposed and unexposed eye in each session.

\section{EXPERIMENT 2 Method}

Subjects

The subjects were the same as those in Experiment 1.

\begin{abstract}
Apparatus
Testing. A pattern consisting of black and white stripes at $4 \mathrm{cycles} / \mathrm{deg}$ was used to assess perception of the vertical. This pattern covered a circular disk which was viewed at a distance of $37.5 \mathrm{~cm}$ and was the only object visible to the subject during testing. The disk was housed within an opaque cylinder into which the subject looked; to change the orientation of the grating, the subject rotated the cylinder by hand. A pointer mounted on the outside of the cylinder permitted the experimenter to record its orientation with respect to a fixed protractor.
\end{abstract}

Exposure. A set of goggles was used to provide monocular exposure with an optically rotated visual field approximately $15^{\circ}$ in diameter as the subject walked about. The line of sight of the viewing eye was interrupted with a set of first-surface mirrors, oriented so as to rotate the field $20^{\circ}$ clockwise when the right eye was exposed and $20^{\circ}$ counterclockwise when the left eye was exposed. The nonviewing eye was occluded.

\section{Procedure}

Each experimental session consisted of a test, exposure, and a retest. All testing was conducted in a darkened room. The orientation of the grating at the start of each trial was adjusted by the experimenter to a position between $40^{\circ}$ clockwise and $40^{\circ}$ counterclockwise from vertical, according to a fixed random sequence. The subjeet could not see the disk while the starting position of the grating was being adjusted. For each trial, the disk was transilluminated for $2 \mathrm{sec}$ while the subject rotated the cylinder to set the grating to apparent vertical. Four binocular practice trials preceded monocular test trials. A soft eye patch was worn over the eye not used. Each eye was used for eight alternate test trials.

Upon completion of preexposure testing, the subject wore the goggles providing monocular view of a rotated field for $30 \mathrm{~min}$ while walking through a series of corridors in our laboratory. Following this exposure, the goggles were removed and 16 postexposure test trials were administered, using the same procedure as in preexposure testing. Adaptation to an optically rotated field produces an apparent counterrotation of the visual field. The first trial was given the subject using the eye which had not been exposed with rotated input, and the eyes were used alternatively for subsequent trials. One week later, the entire procedure was repeated with the fellow eye exposed with optical rotation of the visual field. The session in which dominant and nondominant eyes were exposed was counterbalanced among subjects within each subject group.

\section{Results \\ Variability of Settings}

For each subject, the variance of settings mediated by each eye was computed for both pre-and postexposure tests within each experimental session. Exposed and unexposed eyes did not differ in variance, nor did pre- and postexposure settings mediated by each eye ( $\mathrm{df}=7,7$; criterion values for rejecting the null hypothesis at the .05 level are: $\mathrm{F}<.3$ or $\mathrm{F}>3.8$ ). Therefore, a pooled variance was computed for each subject $\left(s_{p}\right)$ for each session. Mean value of $s_{p}$ was $1.0^{\circ}$; intersession and intersubject differences were not significantly different from zero ( $\mathrm{df}=28,28$; criterion values for rejecting the null hypothesis at the .05 level are: $F<.6$ or $\mathrm{F}>1.8$ ).

\section{Differences Between Pre- and Postexposure Settings}

For each session, for each eye, the mean of preexposure settings was compared with the mean of postexposure settings. For subjects in each group, the mean shift in settings mediated by each eye (dominant or nondominant) for each condition (exposed or unexposed) is presented in Table 2. Positive values represent shifts compensatory for the effect induced by exposure with rotated input. Magnitude of differences was evaluated with $t$ tests (df $=18$; criterion values for rejecting the null hypothesis at the .05 level are $|t|>1.7$ ).

\section{Normal Orthotropes}

For all subjects, exposure of either the dominant or nondominant eye produced compensatory shifts in settings mediated by that eye. There was no difference between dominant and nondominant eye in magnitude of adjustment to rotated input [sign test $\mathrm{r}(8)=4, \mathrm{p}<.25]$. In both cases, the compensatory effect transferred to settings mediated by the unexposed eye with no loss in magnitude [sign test $r(8)=3, p<.25]$.

\section{Strabismic Alternating Suppressors}

Exposure of either the dominant or nondominant eye with optical rotation of input produced compensatory shifts in settings mediated by that eye. Shifts following exposure of the dominant eye did not differ in magnitude from those following exposure of the nondominant eye [sign test $\mathrm{r}(8)=4$, $p<.25]$. In both cases, settings of the vertical mediated by the unexposed eye failed to show the effects of adaptation.

\section{Constant Suppressors (Strabismic Constant Suppressors and Amblyopic Orthotropes}

Following exposure of the dominant eye, settings mediated by either the exposed or unexposed eye

Table 2 Adaptation and Interocular Transfer

\begin{tabular}{lccccr}
\hline & \multicolumn{2}{c}{$\begin{array}{c}\text { Exposed } \\
\text { Eye }\end{array}$} & \multicolumn{2}{c}{$\begin{array}{c}\text { Nonexposed } \\
\text { Eye }\end{array}$} \\
\cline { 2 - 4 } \cline { 5 - 6 } & $\mathrm{D}$ & $\mathrm{ND}$ & $\mathrm{D}$ & $\mathrm{ND}$ \\
\hline & \multicolumn{4}{c}{ Orthotropes } \\
Normal & 4.2 & 3.8 & 3.5 & 3.8 \\
Amblyopic & 3.9 & & 0.2 & 0.3 & 3.9 \\
& \multicolumn{4}{c}{ Strabismics } \\
Alternating Suppressors & 4.4 & & 4.3 & 0.3 & -0.1 \\
Constant Suppressors & 3.9 & & 0.4 & 0.3 & 3.5 \\
\hline
\end{tabular}

Note-Values are expressed as the mean difference (in degrees) between pre-and postexposure settings of the vertical. $D=d o m$ inant; $N D=$ nondominant. 
showed compensation. Shifts in settings mediated by the unexposed eye and those mediated by the exposed eye did not differ in magnitude [sign test $\mathrm{r}(12)=6$, $p<.25]$. In these subjects, exposure of the nondominant eye did not produce adaptation; postexposure settings were not significantly different from preexposure settings.

\section{Discussion}

Orthotropes with normal binocularity of vision adapted to exposure of either eye with rotated input. In agreement with previous reports (Ebenholtz, 1967; Mack \& Chityat, 1970), these subjects showed a compensatory shift in settings of the vertical when tested using either the exposed or the unexposed eye. Alternating suppressors also adapted to exposure of either eye with rotated input. However, in contrast to normal orthotropes, they failed to transfer compensation to settings mediated by the unexposed eye. Finally, in constant suppressors, exposure of the dominant eye with rotated input was followed by an alteration in settings mediated by either that eye or the unexposed eye. In these subjects (both strabismic constant suppressors and amblyopic orthotropes), exposure of the normally suppressed eye with rotated input did not result in a significant shift in settings of the vertical. These results parallel those of Experiment 1 with another technique used to modify input and to assess adaptation.

\section{GENERAL DISCUSSION}

A study of visually guided reaching had demonstrated that strabismic constant suppressors localize a target at two different points in visual space, depending upon the eye of view (Mann, Hein, \& Diamond, 1979). Strabismic alternating suppressors, like orthotropes, did not display this anomaly. These differences were attributed to the developmental history of each group. We hypothesized that constant suppression of vision in one eye enforces acquisition of a representation of visual space which is monocular. That is, the representation incorporates the correspondence between object direction, orbital posture, and retinal locus of images with respect to the dominant eye alone. It was suggested that strabismic alternators acquire two separate representations of visual space, each related to postural information from one eye. Persons with normal binocularity of vision were described as acquiring a single representation of visual space, incorporating postural information from both eyes.

The present study examined the implications of this account for the adjustment of coordination to optical transformations of input. A first experiment showed that persons with normal binocularity of vision adapted to prismatic displacement of input with either eye and transferred the adjustment to reaches mediated by the unexposed eye. Alternating suppressors adapted with either eye but showed no interocular transfer. Constant suppressors adapted only when the dominant eye was exposed. The altered coordination transferred to reaches mediated by the normally suppressed eye. A second experiment replicated this pattern of results for adaptation to optical rotation of the visual field, assessed by a postexposure shift in the apparent vertical.

These results are consistent with our account of the way in which visual space is represented in the three groups of subjects. Adaptation with exposure of either eye and interocular transfer of adaptation is consistent with a single representation of visual space common to both eyes, in persons with normal binocularity of vision. Adaptation with either eye and failure to show interocular transfer of adaptation is consistent with two independent representations of visual space in persons with alternating monocular suppression. Failure of adaptation with exposure of a constantly suppressed eye is consistent with lack of a representation of visual space incorporating postural information from that eye, in both strabismic and orthotropic constant suppressors. In these subjects, adaptation with the dominant eye and transfer to coordinations mediated by the normally suppressed eye is consistent with the existence of a representation of visual space based on the dominant eye alone, perforce utilized when behaviors are mediated by either eye.

All constant suppressors appeared to be able to use the normally suppressed eye; the variance of target markings with that eye did not exceed that for the eye that is consistently in use. However, the representation of visual space used to localize targets presented to the normally suppressed eye could not readily be modified by exposure of that eye. It is possible that a longer period of exposure of the normally suppressed eye would support adaptation. If this occurred, we would not expect the alteration to be transferred to coordinations mediated by the dominant eye. Instead, we would expect the two eyes to be independent, that is, the behavior to follow the pattern demonstrated for alternating suppressors. Observation of the localizing behavior of a group of strabismic constant suppressors who had undergone therapeutic occlusion of the dominant eye early in life supports this conjecture (Mann, Hein, \& Diamond, 1979). In these subjects, enforced monocular exposure of the nondominant eye, although it had not prevented constant suppression, appeared to mitigate against reliance on the posture of the dominant eye in localizing targets presented to the normally suppressed eye.

The issue of the possible neuroanatomic basis for these effects can be raised. Our model relates 
aspects of visuomotor behavior to the pattern of use of the eyes during early development. Other investigators have presented evidence that anomalies of binocular vision are associated with a reduction in the complement of binocular units in visual cortex (Mitchell, Reardon, \& Muir, 1975; Mitchell \& Ware, 1974; Movshon, Chambers, \& Blakemore, 1972; Wade, 1976; Ware \& Mitchell, 1974). A deficiency in stereopsis and a failure to display interocular transfer of certain figural aftereffects (Coltheart, 1971,1973 ) have been attributed to this reduction. Both behavioral deficiencies have been confirmed by us for the strabismics and for the orthotropic constant suppressors who took part in the present study (Mann, 1978).

It does not appear, however, that binocularity of units in primary visual cortex would be relevant to the visuomotor patterns we have investigated here. All strabismics (as well as nonstrabismic amblyopes) presumably are deficient in binocularity of cortical neurons. We have differentiated strabismic alternators and strabismic constant suppressors in terms of the way in which visual space is represented for each. While our studies have not suggested at what level of the visual system these groups might diverge, a representation of visual space is acquired through a process which utilizes motor-visual feedback (Hein \& Diamond, 1972; Held \& Hein, 1963). Therefore, a level where visual and motor information are integrated would appear to be plausible.

\section{REFERENCES}

Bauer, J. A., Woods, G. D., \& Held, R. A device for rapid recording of positional responses in two dimensions. Behavior Research Methods and Instrumentation, 1969, 4, 157-159.

Burian, H. M., \& von Noorden, G. K. Binocular vision and ocular motility. St. Lou is: Mosby, 1974.

Coltheart, M. Visual feature analyzers and after-effects of tilt and curvature. Psychological Review, 1971, 78, 114-121.

Coltheart, M. Colour-specificity and monocularity in the visual cortex. Vision Research, 1973, 13, 2595-2598.

Crawshaw, M., \& Craske, B. Oculomotor adaptation to prisms: Complete transfer between the eyes. British Journal of Psychology, 1976, 67, 475-478.

DEWs, P., \& WiEsel, T. Consequences of monocular deprivation on visual behavior in cats. Journal of Physiology, 1970, 187, 437-445.

Ebenholtz, S. M. Transfer of adaptation as a function of interpolated optical tilt to the ipsilateral and contralateral eye. Journal of Experimental Psychology, 1967, 73, 263-267.

GANz, L., \& Firch, M. The effects of visual deprivation on perceptual behavior in kittens. Experimental Neurology, 1968, 22, 638-660.

Hajos, A., \& RitTer, M. Experiments to the problem of interocular transfer. Acta Psychologica, 1965, 24, 81-90.

HANSEN, A. K. "After-image transfer test" in anomalous retinal correspondence. American Medical Association Archives of Ophthalmology, 1954, 52, 369-374.
Hein, A., \& Diamond, R. M. Contrasting development of visually triggered and guided movements with respect to interocular and interlimb equivalence. Joumal of Comparative and Physiological Psychology, 1971, 76, 219-224.

HeIn, A., \& Diamond, R. Locomotory space as a pre-requisite for acquiring visually-guided reaching in kittens. Journal of Comparative and Physiological Psychology, 1972, 81, 394-398.

HeIN, A., HELD, R., \& GowER, E. Development and segmentation of visually controlled movement by selective exposure during rearing. Journal of Comparative and Physiological Psychology, 1970, 73, 181-187.

HeLD, R., \& Bossom, J. Neonatal deprivation and adult rearrangement: Complementary techniques for analyzing plastic sensorymotor coordinations. Journal of Comparative and Physiological Psychology, 1961, 54, 33-37.

HELD, R., \& GotTlieb, N. Technique for studying adaptation to disarranged hand-eye coordination. Perceptual and Motor Skills, $1958,8,83-86$.

Held, R., \& HeIN, A. Movement-produced stimulation in the development of visually guided behavior. Journal of Comparative and Physiological Psychology, 1963, 56, 872-876.

Held, R., \& Mikaelian, H. Two types of adaptation to an optically-rotated visual field. American Journal of Physiology, $1964,77,257-263$.

MAck, A., \& Chityat, D. Eye-dependent and disparity adaptation to opposite visual-field rotations. American Journal of Physiology, 1970, 83, 352-371.

MANN, V. Separate loci for mediation of tilt and spiral motion after-effects. Investigative Ophthalmology and Visual Science, 1978, 17, 903-909.

Mann, V., Hein, A., \& Diamond, R. Localization of targets by strabismic subjects: Contrasting patterns in constant and alternating suppressors. Perception \& Psychophysics, 1979, 25, 29-34.

Mitchell, D. E., Reardon, J., \& Muir, D. W. Interocular transfer of the motion after-effect in normal and stereoblind observers. Experimental Brain Research, 1975, 22, 163-173.

Mrtchell, D. E., \& W ARE, C. Interocular transfer of a visual after-effect in normal and stereoblind humans. Journal of Physiology, 1974, 236, 707-721.

Movshon, J. A., Chambers, B. E. I., \& Blakemore, C. Interocular transfer in normal humans and those who lack stereopsis. Perception, 1972, 1, 483-490.

Ono, H., Wilkinson, A., Muter, P., \& Mitson, L. Apparent movement and change in perceived location of a stimulus produced by a change in accommodative vergence. Perception \& Psychophysics, 1972, 12, 187-192.

ReINECKE, R. D., \& Miller, D. Strabismus. New York: Appleton-Century-Croft, 1966.

Reinecke, R. D., \& Simon, K. A new stereoscopic test for amblyopic screening. American Journal of Ophthalmology, 1974, 78, 714-721.

Riesen, A. H. Plasticity of behavior. In H. Harlow \& C. Woolsey (Eds.), Biological and Biochemical Bases of Behavior. Madison: University of Wisconsin Press, 1958. Pp. 425-450.

Romano, P. E., \& von Noorden, C. K. Limitations of cover test in detecting strabismus. American Journal of Ophthalmology, $1971,72,10-21$

WADE, N. J. On interocular transfer of the movement after-effect in individuals with and without binocular vision. Perception, $1976,5,113-118$.

WARE, C., \& Mrtchell, D. E. On interocular transfer of various after-effects in normal and stereoblind observers. Vision Research, 1974, 14, 731-734.

(Received for publication May 2, 1978; revision accepted September 29, 1978.) 